\title{
What can be learned from white matter alterations in antisocial girls
}

\author{
Willeke M. Menks' ${ }^{1}$ Christina Stadler' and Nora M. Raschle' \\ 'Department of Child and Adolescent Psychiatry, University of Basel, Psychiatric University Hospital Basel, Switzerland.
}

\section{Article Info}

\section{Article Notes}

Received: June 17, 2017

Accepted: July 31, 2017

\section{*Correspondence:}

Dr. Willeke Menks, PhD

Department of Child and Adolescent Psychiatry (KJPK)

Psychiatric University Clinics Basel (UPK)

Schanzenstrasse 13, CH-4056 Basel, Switzerland

Tel. +41612658976

Fax +4161265 8961

(c) 2017 Menks WM \& Raschle NM. This article is distributed under the terms of the Creative Commons Attribution 4.0 International License

\section{Keywords}

Antisocial behavior

Conduct disorder

Diffusion tensor imaging

Pediatric neuroimaging

Gender

White brain matter

\section{ABSTRACT}

Antisocial behavior in youths constitutes a major public health problem worldwide. Conduct disorder is a severe variant of antisocial behavior with higher prevalence rates for boys (12\%) as opposed to girls (7\%). A better understanding of the underlying neurobiological mechanisms of conduct disorder is warranted to improve identification, diagnosis, or treatment. Functional and structural neuroimaging studies have indicated several key brain regions within the limbic system and prefrontal cortex that are altered in youths with conduct disorder. Examining the structural connectivity, i.e. white matter fiber tracts connecting these brain areas, may further inform about the underlying neural mechanisms. Diffusion tensor imaging (DTI) is a non-invasive technique that can evaluate the white matter integrity of fiber tracts throughout the brain. To date, DTI studies have found several white matter tracts that are altered in youths with conduct disorder. However, a majority of these studies have focused on male or mixed-gender groups, and only a few studies have specifically investigated white matter alterations in girls with conduct disorder. Ultimately, studies that directly compare boys and girls with conduct disorder are necessary to identify possible sexual dimorphic neural alterations and developmental trajectories of conduct disorder in youths.

\section{Introduction}

Antisocial behavior in children and adolescents is associated with significant societal, clinical, and economic consequences and is therefore considered a major public health burden worldwide. Youths with severe aggressive and antisocial behavior outside of the age-appropriate norm qualify for a diagnosis of conduct disorder. Conduct disorder is characterized by a repetitive and persistent pattern of behavior in which the basic rights of others or societal norms or rules are violated ${ }^{1}$. The life time prevalence of conduct disorder is estimated to be around $7 \%$ and $12 \%$, for girls and boys respectively ${ }^{2}$. Furthermore, conduct disorder is thought to be very heterogeneous and several subtypes have been suggested (e.g. with or without callous-unemotional traits ${ }^{3}$ ). Particularly youths with severe subtypes of conduct disorder are at higher risk for persistent antisocial behavior and criminality in adulthood, and thus at great risk for developing antisocial personality disorder later in life ${ }^{1,4}$. Previous studies have suggested that an increased understanding of the neurobiological basis of conduct disorder and its subtypes in youths may increase the modest treatment success of current intervention methods ${ }^{5}$. So far, research studies using functional and structural neuroimaging techniques have identified dysfunctions and structural alterations within a set of cortical and subcortical brain regions. Cortical brain regions that are often identified as being altered in conduct disorder by voxel-based approaches include gray matter structures of the limbic system (e.g. amygdala, insula, and cingulate cortex) and 
prefrontal cortex ${ }^{6-12}$. The limbic system is important for various processes in human social behavior that are often impaired in youths with conduct disorder, such as emotion processing and regulation ${ }^{9}$. The prefrontal cortex (PFC) plays a significant role in cognitive control by means of attention and decision-making over the simple and more automatic behaviors ${ }^{13}$. The amygdala and prefrontal cortex are bi-directionally interconnected through white matter tracts of the prefrontal-limbic circuitry ${ }^{14}$. Investigating the functional and structural connectivity of this circuitry could give more insight in the etiology of conduct disorder ${ }^{15-17}$.

\section{Brain connectivity in conduct disorder}

In line with individual reports, meta-analyses have summarized that brain regions commonly affected in conduct disorder are part of specific neural networks, specifically the emotion processing and regulation network (see figure 1$)^{18,19}$. Brain areas within those networks are functionally and structurally interconnected with one another by anatomical white matter tracts consisting of abundant thin myelinated axons. Thus far, studies investigating functional brain connectivity in youths with severe antisocial behavior have found a reduced functional connectivity between the amygdala and two regions of the prefrontal cortex, namely the ventromedial prefrontal cortex $^{7}$ and the orbitofrontal cortex ${ }^{17,20}$. Consequently, researchers have hypothesized that the observed reduced amygdala-PFC connectivity is correlated with abnormal white matter structures in these youths ${ }^{15,21}$. Although functional networks help to identify the neural dynamics between brain areas, it cannot inform about the actual structural architecture. Hence, investigating white matter tracts will be an important step toward understanding the dysfunctional neural interplay and connectivity that contribute to conduct disorder.

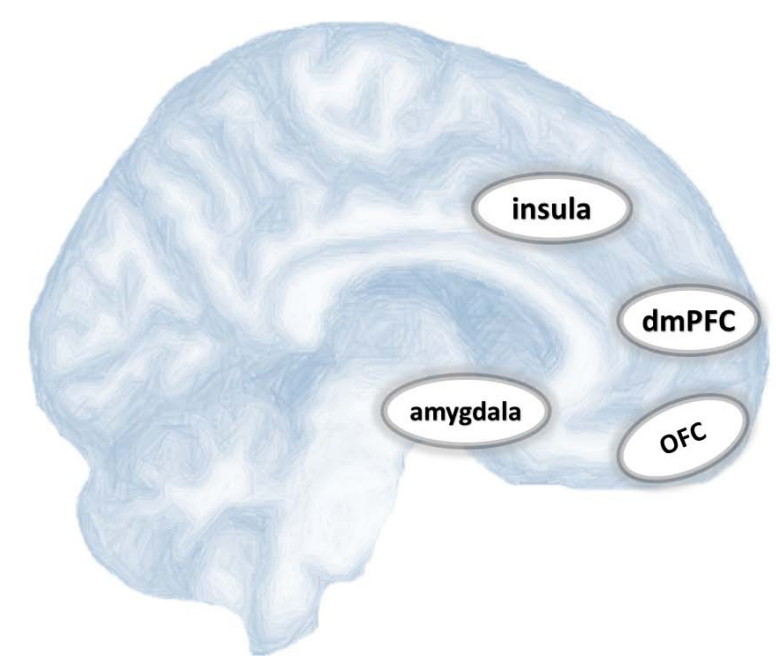

Figure 1. Schematic overview of brain regions commonly affected in conduct disorder: amygdala, insula, dorsomedial prefrontal cortex (dmPFC), and the orbitofrontal cortex (OFC).

\section{Diffusion Tensor Imaging}

A commonly used neuroimaging technique allowing the investigation of the microstructural properties of whitematter is diffusion tensor imaging (DTI). DTI is based on the three-dimensional diffusion of water molecules that is measured through multiple-directional diffusionweighting gradient pulses. Hereby, specific features of this diffusivity are translated into tensors using mathematical equations based on the eigenvectors and eigenvalues within each voxel ${ }^{22}$. The most commonly used tensors to inform about microstructural integrity of white matter tracts are the fractional anisotropy (FA) and mean diffusivity (MD), which measure the anisotropic fraction of diffusivity and the diffusion magnitude respectively ${ }^{23}$. Other tensors occasionally used in DTI studies are axial diffusivity (magnitude of fastest diffusion direction) and radial diffusivity (diffusion magnitude of transverse direction) ${ }^{24}$. In order to analyze group differences, researchers either employ tract-based spatial statistics (TBSS ${ }^{25}$, voxel-based analysis, or fiber tractography.

\section{Altered white matter structures in conduct disorder}

DTI has aided studies investigating white matter structures in children and adolescents with conduct disorder, however, many discrepancies exist between the results of these studies. For example, DTI studies have identified increased fractional anisotropy in the uncinate fasciculus ${ }^{21,26-28}$ and corpus callosum ${ }^{29,30}$ of children and adolescents with conduct problems, while others observed decreased white matter integrity in the same white matter tracts ${ }^{26,31}$, as well as in the corona radiata, superior longitudinal fasciculus, fronto-occipital fasciculus, stria terminalis, and cerebellar peduncle ${ }^{31,32}$. Most studies vary in regard to the precise tract or set of tracts identified, and some do not detect white matter alterations at all ${ }^{20,33,34}$. Thus far, there is no clear picture about the underlying tract-based phenotype of antisocial behavior to date. Similar discrepancies exist when following-up on the relationship of specific DTI tensors in relation to behavioral severity of conduct disorder, such as psychopathic traits, callous unemotional traits, and amount of conduct disorder symptoms ${ }^{31,32,35-37}$. Some identify positive correlations ${ }^{35,37}$, while others reported negative $^{31,32}$ or no correlations at all ${ }^{29}$. These inconsistent findings regarding the direction and location of white mater alterations may result from differences in the analysis approaches used (e.g. TBSS, voxel-based analysis, or fiber tractography), small sample sizes, or group heterogeneity. Especially group heterogeneity is of importance when investigating antisocial behavior. The inclusion of youths can be defined based on different diagnostic criteria; some studies investigated only oppositional defiant disorder, while others focused on conduct disorder or a mixture of both. These heterogeneities were previously mentioned in 
a recent review ${ }^{38}$, however, we suggest another important factor in antisocial behavior, namely sex. So far, most DTI studies have included only male or mixed-gender groups of youths with antisocial behavior. These factors could explain why study results differ in regard to the observed neural alterations.

\section{Sex differences and conduct disorder}

Since most DTI studies have focused solely on male or mix-gender groups, it is unclear whether girls with conduct disorder also show similar white matter alterations as observed in boys ${ }^{21,30-32}$. So far only one study has directly investigated white matter alterations between adolescent boys and girls with conduct disorder and found sex differences for fractional anisotropy, i.e. higher in males within the bilateral uncinate fasciculus ${ }^{28}$. Another study investigating pre-adolescents with conduct problems indicated no sex differences for fractional anisotropy, but did observe a stronger relationship between conduct disorder symptoms and altered white matter integrity (i.e. axial diffusivity) in several tracts, for example the uncinate fasciculus in girls in comparison to boys ${ }^{35}$. Since the uncinate fasciculus interconnects the orbitofrontal cortex and amygdala, this major white matter tract has often been associated with antisocial behavior ${ }^{9,16,17}$. A recent DTI study indicated white matter alterations on a whole-brain level within a homogenous group of girls with conduct disorder ${ }^{29}$. Compared to typically developing girls, girls with conduct disorder had increased FA bilaterally within the body of the corpus callosum expanding towards the right cingulum and left corona radiata, independent of age, intelligence, and attention deficit hyperactivity disorder ${ }^{29}$. These findings align with the study of Zhang and colleagues (2014) who demonstrated FA increases within the body and genu of the corpus callosum of male adolescents with conduct disorder using a TBSS-approach. The corpus callosum has abundant projections to and from the hemispherical cortices, and thus is crucial for interhemispheric communication. The genu and body are subsections of this white matter tract and interconnect parts of the frontal, temporal, and parietal lobes that play an important role in motoric processes, executive functioning, and emotion processing ${ }^{39}$. Alterations of the corpus callosum are also linked to several other neuropsychiatric disorders, such as attention deficit hyperactivity disorder ${ }^{40,41}$, autism ${ }^{42,43}$, Tourette syndrome ${ }^{44}$, or developmental dyslexia ${ }^{45}$. Sex differences in psychiatric disorders ${ }^{46,47}$, e.g. prevalence rates, symptoms, chronicity, and recurrence, are not uncommon and are mostly based on genetic differences. Likewise, there are known sex differences in the behavioral phenotype and developmental trajectories of conduct disorder as revealed by previous epidemiological studies ${ }^{48,49}$. Consequently, this heightens the possibility of observing similar sex-dependent characteristics in the brain. So far, only few neuroimaging studies have directly and indirectly investigated sex differences in youths with conduct disorder on a neural level ${ }^{28,29,50}$, which is likely caused by the lower prevalencerate of conduct disorder in girls ${ }^{2}$. Therefore, an increase in large-site or multicenter studies is needed in order to increase the direct comparison between boys and girls with conduct disorder on a whole-brain level and identify possible sexual dysmorphic neural alterations. Secondly, longitudinal studies allow researchers to investigate the developmental trajectories of conduct disorder in children and adolescents. An increased understanding about sex-differences may lead to the development of future customized intervention programs.

\section{Methodological challenges and gaps in knowledge}

Tensor measurements such as FA or MD inform researchers about the white matter integrity of the fiber tracts. However, it remains difficult to interpret the meaning of these measures on a neuronal or behavioral level. For example, reduced MD may indicate increased myelination or more compact white matter tracts, however, various other factors (e.g. fiber crossings) may play a role as well ${ }^{22}$. Moreover, it remains difficult for even the most advanced programs to determine which brain regions each fiber tract connects to, and even then an altered FA or MD does not automatically indicate an enhanced or diminished activation within that brain region, but merely indicates alterations within the connectivity between brain areas. Therefore, to understand the impact of white matter alterations in conduct disorder, the relationship between fiber tracts and behavior needs further investigation using advanced neuroimaging techniques in both humans and animal models. Also, indirect correlations with healthy participants can inform the scientific field more about factors influencing the development of conduct disorder.

\section{Summary}

Neuroimaging studies have indicated several key brain regions within the limbic system (e.g. amygdala and insula) and prefrontal cortex (e.g. orbitofrontal cortex and ventromedial prefrontal cortex) that display alterations of gray matter volume and brain activity in youths with severe antisocial behavior. These brain regions are interconnected through white matter fiber tracts. Examining these tracts enhances the understanding how altered neural connectivity is linked to conduct disorder. DTI is a useful neuroimaging technique for researchers to non-invasively investigate the white matter integrity of fiber tracts throughout the brain. Nevertheless, to date DTI findings are inconsistent regarding the direction and location of white mater alterations in youths with severe antisocial behavior. These discrepancies are likely caused by the heterogeneity of the samples included in each study. The inclusion of homogeneous or separate samples regarding 
sex and clinical diagnosis is therefore recommended for DTI studies that investigate the relationship between white matter alterations and conduct disorder. Furthermore, an increase in large-scale or multicenter studies using longitudinal approaches enhances the possibility to inform about gender specific developmental characteristics. In this manner, DTI studies can further our knowledge of the underlying neurobiological mechanism of antisocial behavior which can aid the development of effective prevention and intervention programs.

\section{Acknowledgement}

Dr. Menks, Prof. Stadler, and Dr. Raschle are with the University of Basel, Psychiatric University Hospital Basel, Switzerland. This research was supported by a grant from the Swiss National Foundation [105314M_150282]. Dr. Raschle is a recipient of an early career Jacobs Foundation fellowship and received grants of the University of Basel and UPK in Basel (Nachwuchsförderung). Dr. Menks, Prof. Stadler, and Dr. Raschle report no biomedical financial interests or potential conflicts of interest. Prof. Stadler received grants from the European Commission (FP7 funding).

\section{References}

1. American Psychiatric Association. Diagnostic and statistical manual of mental disorders. 5th ed., Washington, DC, American Psychiatric Press.; 2013.

2. Nock MK, Kazdin AE, Hiripi E, et al. Prevalence subtypes and correlates of dsm-iv conduct disorder in the national comorbidity survey replication. Psychol Med. 2006; 36: 699-710.

3. Frick PJ, White SF. Research review The importance of callousunemotional traits for developmental models of aggressive and antisocial behavior. Journal of child psychology and psychiatry and allied disciplines. 2008; 49: 359-375.

4. Lahey BB, Loeber R, Burke JD, et al. Predicting future antisocial personality disorder in males from a clinical assessment in childhood. Journal of Consulting and Clinical Psychology. 2005; 73: 389-399.

5. Woolfenden SR, Williams K, Peat J. Family and parenting interventions for conduct disorder and delinquency: A meta-analysis of randomised controlled trials. Archives of disease in childhood. 2002; 86: 251-256.

6. Finger EC, Marsh AA, Mitchell DG, et al. Abnormal ventromedial prefrontal cortex function in children with psychopathic traits during reversal learning. Arch Gen Psychiatry. 2008; 65: 586-594.

7. Marsh AA, Finger EC, Mitchell DG, et al. Reduced amygdala response to fearful expressions in children and adolescents with callousunemotional traits and disruptive behavior disorders. Am J Psychiatry. 2008; 165: 712-720.

8. Jones AP, Laurens KR, Herba CM, et al. Amygdala hypoactivity to fearful faces in boys with conduct problems and callous-unemotional traits. The American Journal of Psychiatry. 2009; 166: 95-102.

9. Blair RJ. The neurobiology of psychopathic traits in youths. Nat Rev Neurosci. 2013; 14: 786-799.

10. Dolan M. Neurobiological disturbances in callous-unemotional youths. Am J Psychiatry. 2008; 165: 668-670.

11. Sebastian C, Mccrory E, Dadds M, et al. Neural responses to fearful eyes in children with conduct problems and varying levels of callous unemotional traits. Psychological medicine. 2014; 44: 99-109.
12. Sterzer P, Stadler C, Krebs A, et al. Abnormal neural responses to emotional visual stimuli in adolescents with conduct disorder. Biol Psychiatry. 2005; 57: 7-15.

13. Ridderinkhof KR, Van Den Wildenberg WP, Segalowitz SJ, et al Neurocognitive mechanisms of cognitive control: The role of prefrontal cortex in action selection response inhibition performance monitoring and reward-based learning. Brain and cognition. 2004; 56: $129-140$.

14. Amaral DG, Price J. Amygdalo-cortical projections in the monkey macaca fascicularis. Journal of Comparative Neurology. 1984; 230: 465-496.

15. Blair R. The amygdala and ventromedial prefrontal cortex: Functional contributions and dysfunction in psychopathy. Philos Trans R Soc Lond B Biol Sci. 2008 ; 363 :2557-2565.

16. Craig MC, Catani M, Deeley Q et al. Altered connections on the road to psychopathy. Mol Psychiatry. 2009; 14: 946-953, 907.

17. Marsh AA, Finger EC, Fowler KA, et al. Reduced amygdala-orbitofrontal connectivity during moral judgments in youths with disruptive behavior disorders and psychopathic traits. Psychiatry Res. 2011; 194: 279-286.

18. Raschle NM, Menks WM, Fehlbaum LV, et al. Structural and functional alterations in right dorsomedial prefrontal and left insular cortex co-localize in adolescents with aggressive behaviour: An ale metaanalysis. PLoS One. 2015; 10: e0136553.

19. Rogers JC, De Brito SA. Cortical and subcortical gray matter volume in youths with conduct problems: A meta-analysis. JAMA Psychiatry. 2016; 73: 64-72.

20. Finger EC, Marsh A, Blair KS, et al. Impaired functional but preserved structural connectivity in limbic white matter tracts in youth with conduct disorder or oppositional defiant disorder plus psychopathic traits. Psychiatry Res. 2012; 202: 239-244.

21. Passamonti L, Fairchild G, Fornito A, et al. Abnormal anatomical connectivity between the amygdala and orbitofrontal cortex in conduct disorder. PLoS One. 2012; 7: e48789.

22. Beaulieu $\mathrm{C}$. The basis of anisotropic water diffusion in the nervous system a technical review. NMR in Biomedicine. 2002; 15: 435-455.

23. Pierpaoli C, Basser PJ. Toward a quantitative assessment of diffusion anisotropy. Magnetic resonance in Medicine. 1996; 36: 893-906.

24. Song SK, Sun SW, Ramsbottom MJ, et al. Dysmyelination revealed through mri as increased radial (but unchanged axial) diffusion of water. Neuroimage. 2002; 17: 1429-1436.

25. Smith SM, Jenkinson M, Johansen Berg H, et al. Tract-based spatial statistics: Voxelwise analysis of multi-subject diffusion data. Neuroimage. 2006; 31: 1487-1505.

26. Sarkar S, Craig MC, Catani M, et al. Frontotemporal white-matter microstructural abnormalities in adolescents with conduct disorder: A diffusion tensor imaging study. Psychol Med. 2013; 43: 401-411.

27. Sobhani M, Baker L, Martins B, et al. Psychopathic traits modulate microstructural integrity of right uncinate fasciculus in a community population. Neuroimage Clin. 2015; 8: 32-38.

28. Zhang J, Gao J, Shi $\mathrm{H}$, et al. Sex differences of uncinate fasciculus structural connectivity in individuals with conduct disorder. Biomed Res Int. 2014; 2014: 673165.

29. Menks WM, Furger R, Lenz C, et al. Microstructural white matter alterations in the corpus callosum of girls with conduct disorder. J Am Acad Child Adolesc Psychiatry. 2017; 56: 258-265 e251.

30. Zhang J, Zhu X, Wang X, et al. Increased structural connectivity in corpus callosum in adolescent males with conduct disorder. J Am Acad Child Adolesc Psychiatry. 2014; 53: 466-475 e461.

31. Breeden AL, Cardinale EM, Lozier LM, et al. Callous-unemotional traits drive reduced white-matter integrity in youths with conduct problems. Psychol Med. 2015; 45: 3033-3046. 
32. Haney Caron E, Caprihan A, Stevens MC. Dti-measured white matter abnormalities in adolescents with conduct disorder. J Psychiatr Res. 2014; 48: 111-120.

33. Beyer F, Munte TF, Wiechert J, et al. Trait aggressiveness is not related to structural connectivity between orbitofrontal cortex and amygdala. PLoS One. 2014; 9: e101105.

34. Hummer TA, Wang Y, Kronenberger WG, et al. The relationship of brain structure to age and executive functioning in adolescent disruptive behavior disorder. Psychiatry Res. 2015; 231: 210-217.

35. Decety J, Yoder KJ, Lahey BB. Sex differences in abnormal white matter development associated with conduct disorder in children. Psychiatry Res. 2015; 233: 269-277.

36. Yoder KJ, Lahey BB, Decety J. Callous traits in children with and without conduct problems predict reduced connectivity when viewing harm to others. Sci Rep. 2016; 6: 20216.

37. Pape LE, Cohn MD, Caan MW, et al. Psychopathic traits in adolescents are associated with higher structural connectivity. Psychiatry Res. 2015; 233: 474-480.

38. Waller R, Dotterer HL, Murray L, et al. White-matter tract abnormalities and antisocial behavior: A systematic review of diffusion tensor imaging studies across development. NeuroImage Clinical. 2017.

39. Schulte T, Muller Oehring EM. Contribution of callosal connections to the interhemispheric integration of visuomotor and cognitive processes. Neuropsychol Rev. 2010; 20: 174-190.

40. Hynd GW, Semrud Clikeman M, Lorys AR, et al. Corpus callosum morphology in attention deficit-hyperactivity disorder: Morphometric analysis of mri. Journal of Learning Disabilities. 1991; 24: 141-146.

41. Catherine A. Quantitative morphology of the corpus callosum in attention deficit hyperactivity disorder. Am J Psychiatry. 1994; 151 665-669.
42. Vidal CN, Nicolson R, Devito TJ, et al. Mapping corpus callosum deficits in autism: An index of aberrant cortical connectivity. Biological psychiatry. 2006; 60: 218-225.

43. Herbert MR, Ziegler DA, Makris N, et al. Localization of white matter volume increase in autism and developmental language disorder. Annals of neurology. 2004; 55: 530-540.

44. Plessen KJ, Grüner R, Lundervold A, et al. Reduced white matter connectivity in the corpus callosum of children with tourette syndrome. Journal of Child Psychology and Psychiatry. 2006; 47 1013-1022.

45. Hasan KM, Molfese DL, Walimuni IS, et al. Diffusion tensor quantification and cognitive correlates of the macrostructure and microstructure of the corpus callosum in typically developing and dyslexic children. NMR in Biomedicine. 2012; 25: 1263-1270.

46. Baron Cohen S, Knickmeyer RC, Belmonte MK. Sex differences in the brain: Implications for explaining autism. Science. 2005; 310: 819823.

47. Piccinelli M and Wilkinson G. Gender differences in depression. The British Journal of Psychiatry. 2000;177:486-492.

48. Silverthorn P, Frick PJ. Developmental pathways to antisocial behavior: The delayed-onset pathway in girls. Development and psychopathology. 1999; 11: 101-126.

49. Cohen P, Cohen J, Kasen S, et al. An epidemiological study of disorders in late childhood and adolescencei age-and gender-specific prevalence. Journal of child psychology and psychiatry. 1993; 34: 851867.

50. Fairchild G, Hagan CC, Walsh ND, et al. Brain structure abnormalities in adolescent girls with conduct disorder. J Child Psychol Psychiatry. 2013; 54: 86-95. 